\title{
Estresse psicossocial e resiliência: um estudo em profissionais da enfermagem
}

Psychosocial stress and resilience: a study in nursing professionals

Estrés psicosocial y resiliencia: un estudio en profesionales de enfermeira

\section{Andréia Barcellos Teixeira Macedo', Liliana Antoniolli"I, Thayane Martins Dornelles ${ }^{\mathrm{III}}$, Leandro Augusto Hansel ${ }^{\mathrm{IV}}$, Juliana Petri Tavares ${ }^{\mathrm{v}}$, Sônia Beatriz Cocaro de Souzavi}

\begin{abstract}
Resumo: Objetivo: verificar presença de estresse psicossocial e escores de resiliência nos profissionais da enfermagem que cuidam de adultos com germes multirresistentes. Método: estudo transversal, realizado em uma unidade de internação para adultos, em um hospital universitário. A amostra foi intencional, composta por 39 profissionais da enfermagem, com dados coletados de janeiro a maio de 2014 por meio dos questionários Desequilíbrio Esforço-Recompensa e Escore de Resiliência. Resultados: a média de idade foi de 39,2 $\pm 9,0$ anos. Observou-se que 69,23\% (27) dos profissionais apresentaram elevado estresse psicossocial e 56,41\% (22) baixa resiliência. A correlação entre o estresse psicossocial e a resiliência foi de $r=0,3(p=0,001)$. Conclusão: identificouse elevado percentual de profissionais com estresse psicossocial e com baixa resiliência, indicando risco de adoecimento psicoemocional e físico. Este resultado reforça a importância de revisar a organização do trabalho, estimulando a implementação de programas para aumento dos escores de resiliência.
\end{abstract}

Descritores: Saúde do trabalhador; Estresse psicológico; Resiliência psicológica; Equipe de enfermagem

Abstract: Objective: to verify the presence of psychosocial stress and resilience scores in nursing professionals who care for adults with multidrug-resistant germs. Method: cross-sectional study, carried out in an inpatient unit for adults, in a university hospital. The sample was intentional, composed of 39 nursing professionals, with data collected from January to May 2014 through the questionnaire Effort-Reward Imbalance and Resilience Score.

\footnotetext{
I Enfermeira, Doutoranda de Enfermagem, Universidade Federal do Rio Grande do Sul, porto Alegre, Rio Grande do Sul, Brasil; abtmacedo@gmail.com; http://orcid.org/0000-0003-4219-4731

II Enfermeira, Doutoranda de Enfermagem, Universidade Federal do Rio Grande do Sul, porto Alegre, Rio Grande do Sul, Brasil; l.antoniolli@hotmail.com; https://orcid.org/0000-0003-0806-9910

III Enfermeira, Mestre em Enfermagem, Universidade Federal do Rio Grande do Sul, porto Alegre, Rio Grande do Sul, Brasil; thaydornelles@gmail.com; https://orcid.org/0000-0001-7377-7054

IV Enfermeiro, Especialista, Universidade Federal do Rio Grande do Sul, porto Alegre, Rio Grande do Sul, Brasil; lhansel@hcpa.edu.br; https://orcid.org/0000-0002-9580-9085

v Enfermeira, Doutora em Enfermagem, Universidade Federal do Rio Grande do Sul, porto Alegre, Rio Grande do Sul, Brasil; jupetritavares@gmail.com; https://orcid.org/0000-0003-4121-645X

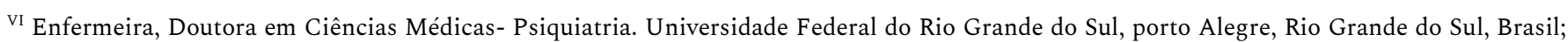
sbcs2001@gmail.com; https://orcid.org/0000-0001-9394-5465
} 
Estresse psicossocial e resiliência: um estudo em profissionais da enfermagem | 2

Results: the mean age was $39.2 \pm 9.0$ years. It was observed that $69.23 \%$ (27) of the professionals had high psychosocial stress and $56.41 \%$ (22) low resilience. The correlation between psychosocial stress and resilience was $\mathrm{r}$ $=0.3(\mathrm{p}=0.001)$. Conclusion: a high percentage of professionals with psychosocial stress and low resilience was identified, indicating a risk of psychoemotional and physical illness. This result reinforces the importance of reviewing the organization of work, encouraging the implementation of programs to increase resilience scores.

Descriptors: Occupational health; Stress, psychological; Resilience psychological; Nursing team

Resumen: Objetivo: verificar la presencia de estrés psicosocial y de resiliencia en profesionales de enfermería que atienden a adultos con gérmenes resistentes a múltiples fármacos. Método: estudio transversal, realizado en una unidad de internación para adultos, en hospital universitario. La muestra fue intencional, compuesta por 39 profesionales, con datos recopilados de enero a mayo de 2014 a través del cuestionario Effort-Reward Imbalance and Resilience Score. Resultados: la edad media fue de 39,2 \pm 9,0 años. Se observó que 69.23\% (27) de los profesionales tenían alto estrés psicosocial y $56.41 \%$ (22) baja resiliencia. La correlación entre estrés psicosocial y resiliencia fue $\mathrm{r}=$ $0.3(\mathrm{p}=0.001)$. Conclusión: se identificó alto porcentaje de profesionales con estrés psicosocial y baja resiliencia, lo que indica riesgo de enfermedad psicoemocional y física. Este resultado refuerza la importancia de revisar la organización del trabajo, alentando la implementación de programas para aumentar los puntajes de resiliencia.

Descriptores: Salud laboral; Estrés psicológico; Resiliencia psicológica; Grupo de enfermería

\section{Introdução}

O estresse figura entre os maiores problemas de saúde e como coadjuvante em várias patologias, como doenças cardíacas, câncer, acidente vascular encefálico, bem como em muitos problemas psiquiátricos, como ansiedade e depressão. O estresse ocupacional está associado à hipertensão, doenças cardiovasculares e à distúrbios psíquicos, os quais podem contribuir para ocorrência de 120.000 mortes por ano na América e estão relacionados à alta taxa de morbidade e elevado custo para tratamento. ${ }^{1}$

O estresse psicossocial refere-se ao estado emocional resultante de uma discrepância entre o grau de exigência do trabalho e os recursos disponíveis para gerenciá-lo, constituindo um fenômeno subjetivo e decorrente da compreensão individual da incapacidade para gerenciar as exigências laborais. Pode sofrer influência dos aspectos da profissão, processo de trabalho, cultura da empresa, bem como pela maneira como o indivíduo consegue lidar com as situações adversas. ${ }^{2}$

Dentre os modelos teóricos que avaliam o estresse psicossocial, destaca-se o Modelo Desequilíbrio Esforço-Recompensa (DER), o qual postula que o trabalhador está em equilíbrio, 
3 | Macedo ABT, Antoniolli L, Dornelles TM, Hansel LA, Tavares JP, Souza SBC

quando percebe que o esforço despendido para a realização das suas atividades é compensado pelas recompensas recebidas por parte do empregador. Também avalia o grau de comprometimento do indivíduo com o trabalho. Quando existe a percepção de baixa recompensa advinda da instituição, o excesso de comprometimento, quando há esforço além dos níveis considerados apropriados, potencializar o risco para estresse psicossocial. ${ }^{3}$ Assim, as experiências cronicamente estressantes são o resultado de um desequilíbrio entre altos esforços e baixas recompensas, somado a um alto nível de comprometimento.

O estresse estimula os indivíduos a buscar estratégias conscientes ou inconscientes para superar com as situações adversas. A habilidade inconsciente do indivíduo em se adaptar ou se reconstruir a partir da adversidade denomina-se resiliência. Sendo assim, a resiliência constitui uma característica dinâmica entre a habilidade do indivíduo diante de um conflito, num determinado contexto e deve ser considerada quando se quer conhecer a relação entre sobrecarga de trabalho e estresse psicossocial. ${ }^{4}$

Atualmente, semelhante ao conceito de estresse, a resiliência pode ser vista de uma forma individual, ocupacional ou organizacional. A resiliência no trabalho é a capacidade de gerenciar o estresse diário do trabalho de forma a permanecer saudável e de aprender e se recuperar com os contratempos inesperados, preparando-se para desafios futuros de forma proativa. ${ }^{5}$ Por outro lado, muitos profissionais de saúde, embora expostos a situações adversas, mostram-se saudáveis e desempenham adequadamente suas funções, prestando serviços de qualidade à população. ${ }^{6}$

O trabalho da enfermagem é caracterizado por estressores decorrentes das cargas físicas e mentais associados a desfechos negativos como abandono da profissão, lesões, adoecimento, absenteísmo, insatisfação no trabalho, prejuízo na qualidade de vida e comprometimento da capacidade para o trabalho. ${ }^{7}$ Uma avaliação realizada nas publicações sobre o tema concluiu que o surgimento do estresse na enfermagem deve ser visto de forma mais ampla, sendo geralmente de origem multifatorial, sofrendo interferência de questões sociais, pessoais, do trabalho, da organização, entre outros. ${ }^{8}$ 
Estresse psicossocial e resiliência: um estudo em profissionais da enfermagem $\mid 4$

Para proteger os trabalhadores da enfermagem do risco de adoecimento pelo estresse ocupacional, estudiosos da área sugerem a implantação de programas de redução do estresse e estratégias de promoção de resiliência, fortalecendo recursos internos nestes profissionais e ambientes saudáveis de trabalho. Assim, mapear a resiliência poderá auxiliar a identificar características de proteção na equipe de enfermagem, frente às condições adversas de trabalho. ${ }^{9}$

O investimento em tecnologias para a recuperação dos doentes e em programas de qualidade assistencial vêm modificando o processo de trabalho na enfermagem. O atendimento a pacientes com germes multirresistentes (GMR), por exemplo, transformou a maneira de cuidar. Por ser um problema relativamente novo no cenário nacional, as instituições de saúde ainda estão buscando formas de organizar o trabalho para o atendimento desta clientela, de modo a manter todas as medidas de barreira epidemiológica preconizadas, preservando a segurança de todos os pacientes e poucas instituições possuem um setor específico para internação e tratamento de pacientes portadores de GMR. ${ }^{10-11}$

Uma instituição pública da região sul do Brasil apostou na implantação de uma unidade de internação específica para o atendimento de pacientes clínicos e cirúrgicos com GMR. Os resultados demonstraram um decréscimo importante na incidência de bactérias resistentes, principalmente nas infecções causadas pelo Enterococcus spp resistente à vancomicina e Pseudomonas aeruginosa resistente aos carbapenêmicos. ${ }^{10}$ Entretanto, observa-se que o atendimento dos pacientes com GMR acarreta sobrecarga no trabalho dos profissionais da enfermagem, pois o tratamento requer a execução de um número elevado de atividades no seu turno de trabalho.

Usualmente são pacientes com elevado nível de complexidade assistencial, necessitando da enfermagem para suprir todas as suas necessidades corporais. ${ }^{12}$ A utilização de barreiras de proteção para a redução do risco de transmissão cruzada dentro da instituição hospitalar implica em tempo para vestir e retirar luvas e avental a cada contato com os pacientes e cuidado rigoroso com higiene de mãos, além de auditorias constantes. Após a implantação da unidade 
se identificou aumento no absenteísmo da equipe de enfermagem do setor, fato que gerou o interesse por este estudo.

Frente ao exposto, esta investigação parte da seguinte questão de pesquisa: Existe estresse psicossocial nos profissionais da enfermagem que atendem pacientes com GMR e como se apresentam os escores de resiliência? $\mathrm{O}$ objetivo foi verificar a presença de estresse psicossocial e mensurar os escores de resiliência nos profissionais da enfermagem que cuidam de adultos com GMR em um hospital universitário.

\section{Método}

Este artigo foi extraído da pesquisa “Avaliação da carga de trabalho, estresse psicossocial e resiliência nos profissionais de enfermagem em uma unidade de internação para portadores de germes multirresistentes” e tratou-se de uma dissertação. ${ }^{13}$

Estudo com delineamento quantitativo e observacional, do tipo transversal, realizado em uma unidade de internação para adultos com GMR de um hospital universitário da região Sul do Brasil. Trata-se de um setor com 34 leitos, disponíveis para receber pacientes clínicos, cirúrgicos e de especialidades médicas, com GMR. A triagem dos pacientes a internarem neste setor, assim como a liberação das medidas de precaução, são atividades realizadas pela Comissão de Controle de Infecção Hospitalar (CCIH) da instituição. São pacientes oriundos da Unidade de Tratamento Intensivo (UTI), da Emergência e de outras unidades de internação clínicas ou cirúrgicas da própria instituição.

A população de interesse foi a equipe de enfermagem exclusiva do setor, composta por 10 enfermeiros, 15 auxiliares e 21 técnicos de enfermagem. Foram incluídos todos os profissionais da enfermagem da unidade e excluídos os indivíduos que possuíam retorno há menos de duas semanas de afastamento prolongado e os que se encontravam em período probatório de 90 dias. Entende-se como afastamento prolongado o período referente à 
Estresse psicossocial e resiliência: um estudo em profissionais da enfermagem | 6

concessão de benefício por doença do Instituto Nacional de Seguridade Social (INSS) e Licença Gestação (LG).

Excluiu-se três profissionais que haviam retornado de INSS, um de LG e três que estavam em período probatório, constituindo uma amostra com 39 profissionais, com 10 enfermeiros, 13 auxiliares e 16 técnicos, caracterizando uma amostra intencional. Optou-se por contabilizar técnicos e auxiliares de enfermagem como uma única categoria pelo fato de desenvolverem atividades equivalentes.

A coleta de dados ocorreu de janeiro a maio de 2014, com a utilização do Questionário de Desequilíbrio Esforço-Recompensa (DER) e do Escore de Resiliência. Coletou-se informações pessoais e da atividade profissional a partir de instrumento de caracterização dos sujeitos construído para a pesquisa, incluindo sexo, idade, escolaridade, situação conjugal, número de filhos, tempo na enfermagem, no setor, turno de trabalho e vínculo empregatício.

A presença do estresse psicossocial foi avaliado por meio da versão curta do questionário de DER, constituída por 16 questões, que definem medidas em três dimensões: Esforço (pressão de tempo, interrupções, responsabilidades, pressão para fazer horas extras, trabalho fisicamente exigente e aumento de demanda); Recompensa (gratificação financeira, salário e esforço; expectativa de estima e reconhecimento; respeito, apoio adequado, tratamento inadequado; segurança e oportunidades de carreira - expectativa de promoção e ascensão, mudanças indesejáveis, insegurança no trabalho e inconsistência do status ocupacional); e excesso de comprometimento com o trabalho (grupo de atitudes, comportamentos e emoções que refletem um esforço além dos níveis considerados apropriados para ser aprovado e valorizado pelos outros).

Para cada questão é apresentada uma escala Likert, sendo que cada dimensão resulta em um escore. O desequilíbrio entre esforço-recompensa é demonstrado quando aplicada a equação (e/r x c), sendo "e" a soma dos itens de esforço, "r" a soma dos itens de recompensa e "c" o fator de correção. Valores maiores a 1 indicam desequilíbrio entre o esforço e recompensa. O excesso de comprometimento com o trabalho é considerado um fator que pode interagir com o 
7 | Macedo ABT, Antoniolli L, Dornelles TM, Hansel LA, Tavares JP, Souza SBC

desequilíbrio esforço-recompensa, potencializando seus efeitos nocivos à saúde e ao bem-estar. ${ }^{3}$ Os resultados das dimensões esforço e recompensa foram dicotomizados em alto ou baixo a partir da mediana e o excesso de comprometimento em tercis.

O Escore de Resiliência é usado para medir níveis de adaptação psicossocial positiva em face de eventos de vida importantes. Possui 25 itens descritos de forma positiva com resposta tipo likert variando de 1 (discordo totalmente) a 7 (concordo totalmente). A soma dos itens resulta em escores que oscilam de 25 a 175 pontos. O autor sugere que a pontuação encontrada seja dividida em resiliência muito baixa(25 a 100), baixa(101 a 115), moderadamente baixa (116 a 130), moderadamente alta (131 a 145), alta (145 a 160) e muito alta (161 a 175). ${ }^{14}$ Por orientação de um profissional da estatística, neste estudo será considerada baixa resiliência valores $\leq 130$ pontos e alta resiliência $\geq 131$ pontos

Os dados foram agrupados em planilhas com auxílio do pacote estatístico Statistical Package for the Social Sciences (SPSS), versão 20.0. As variáveis foram analisadas individualmente por meio de estatística descritiva, com medidas de tendência central e dispersão, frequência absoluta e relativa. Para a avaliação da relação das variáveis contínuas foi usado o teste $\mathrm{T}$ independente e a relação entre os resultados encontrados nas escalas foi avaliada por meio do Coeficiente de Pearson. Valores de "p" menores que 0,05 foram considerados estatisticamente significativos.

Por tratar-se de uma pesquisa com seres humanos, o estudo foi desenvolvido atendendo aos critérios da Resolução $\mathrm{n}^{\circ} 466 / 2012$ do Conselho Nacional de Saúde. O projeto foi aprovado pelo Comitê de Ética em Pesquisa no dia 26 de dezembro de 2012, sob protocolo número 120165.

\section{Resultados}

Dos 39 participantes, 10 (25,6\%) eram enfermeiros e 29 (74,4\%) eram auxiliares e técnicos de enfermagem. As características sociodemográficas dos trabalhadores se encontram na Tabela 1: 
Estresse psicossocial e resiliência: um estudo em profissionais da enfermagem $\mid 8$

Tabela 1 - Características sociodemográficas e de saúde dos trabalhadores de enfermagem. Porto Alegre, RS, Brasil, 2014.

\begin{tabular}{|c|c|}
\hline Variáveis & $\mathbf{N}=39$ \\
\hline Idade $^{*}$ (anos) & $39,2 \pm 9,0$ \\
\hline Sexo Feminino** & $32(82,1)$ \\
\hline \multicolumn{2}{|l|}{ Escolaridade ${ }^{* *}$} \\
\hline Ensino Superior & $13(33,3)$ \\
\hline Ensino Superior incompleto & $04(10,3)$ \\
\hline Ensino Médio & $22(56,4)$ \\
\hline \multicolumn{2}{|l|}{ Situação conjugal ${ }^{* *}$} \\
\hline Casado/companheiro (a) & $26(66,7)$ \\
\hline Solteiro (a) & $13(33,3)$ \\
\hline \multicolumn{2}{|l|}{ Número de filhos** } \\
\hline Sem filhos & $11(28,2)$ \\
\hline Com 1 filho & $12(30,8)$ \\
\hline Com 2 filhos & $11(28,2)$ \\
\hline Com 3 filhos ou mais & $05(12,8)$ \\
\hline Tempo na enfermagem ${ }^{*}(\operatorname{anos})$ & $12,0 \pm 8,1$ \\
\hline Tempo no setor ${ }^{*}$ anos $)$ & $8,32 \pm 7,44$ \\
\hline \multicolumn{2}{|l|}{ Vínculo empregatício** } \\
\hline Apenas 1 & $37(94,9 \%)$ \\
\hline Turno de trabalho ${ }^{* *}$ & $22(56,3 \%)$ \\
\hline diurno & \\
\hline
\end{tabular}

Fonte: Dados da pesquisa. Porto Alegre, 2014

Nota: *Média \pm desvio-padrão. ${ }^{* *}$ Números expressos em $\mathrm{n}(\%)$.

Observou-se que 29 (74,36\%) dos profissionais percebiam alto esforço na realização do trabalho e 23 (59\%) baixa recompensa. O estresse psicossocial foi identificado em $27(69,23 \%)$ profissionais, dos quais 9 (90\%) eram enfermeiros e 18 (62,07\%) eram auxiliares/técnicos de enfermagem, conforme apresentado na Figura 1: 
Figura 1 - Estresse Psicossocial nos profissionais da enfermagem que assistem pacientes com germes multirresistentes em um hospital universitário. Porto Alegre, RS, Brasil, 2014.

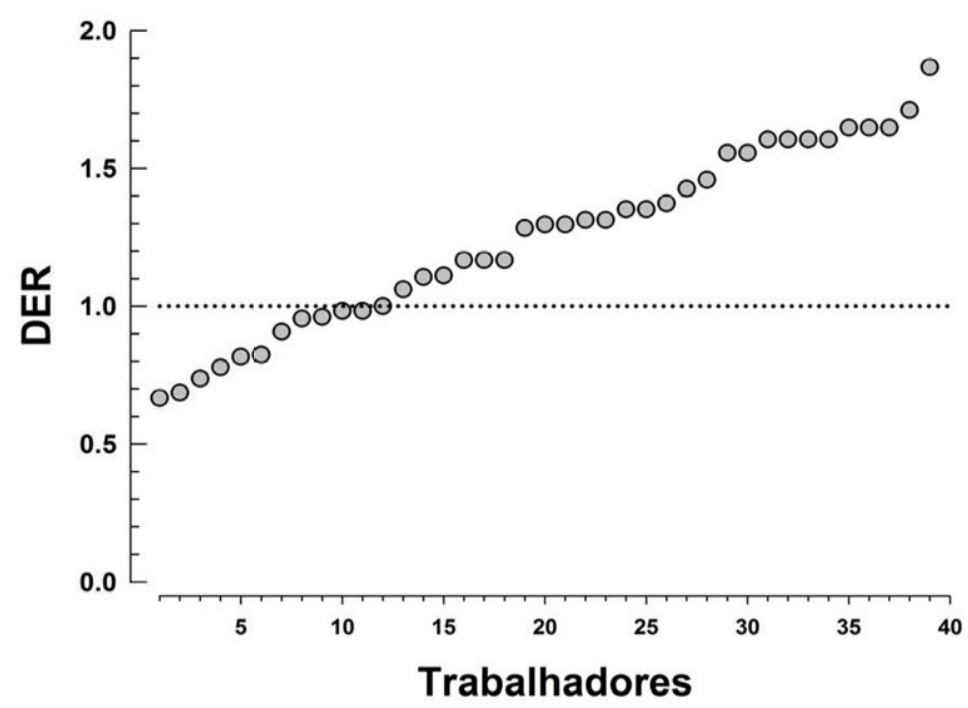

Em relação ao domínio excesso de comprometimento, a média foi de 12,28ะ2,05 pontos. Observou-se baixo comprometimento em 13(33,3\%) profissionais, médio em 19(48,7\%) e alto em $7(17,9 \%)$ com $\mathrm{p}=0,063$. A relação do domínio excesso de comprometimento com o estresse psicossocial foi $0,54(\mathrm{p}<0,001)$. Já a relação do excesso de comprometimento com o domínio esforço foi de $r=0,59(p<0,001)$ e com a recompensa foi de $r=-0,17(p=0,584)$.

A avaliação do estresse psicossocial entre as categorias profissionais demonstrou que não houve diferença estatisticamente significativa entre enfermeiros e auxiliares/técnicos $(\mathrm{p}=0,650)$. Em relação ao turno de trabalho, observou-se maior estresse psicossocial nos trabalhadores do turno da noite $(1,39 \pm 0,25)$ em relação aos do diurno $(1,12 \pm 0,35)(\mathrm{p}=0,012)$.

A média encontrada no escore de Resiliência foi de $132 \pm 15,9$ pontos, com valores entre 113 e 172 pontos. Constatou-se que $22(56,41 \%)$ profissionais apresentaram baixa resiliência, conforme apresentado na Figura 2: 
Figura 2 - Escore de Resiliência nos profissionais da enfermagem que assistem pacientes com de germes multirresistentes em um hospital universitário. Porto Alegre, RS, Brasil, 2014.



A comparação entre categorias profissionais demonstrou que não houve diferença estatisticamente significativa entre enfermeiros e auxiliares/técnicos quanto ao escore de resiliência ( $p=0,578)$, o mesmo ocorrendo por turno de trabalho, em que não se observou diferença estatisticamente significativa nos profissionais do diurno e do noturno ( $\mathrm{p}=0,127)$. A correlação entre estresse psicossocial e o escore de resiliência nos profissionais estudados foi de 0,3 ( $\mathrm{p}=0,001)$.

\section{Discussão}

Verificou-se que a maioria dos profissionais apresentam estresse psicossocial, alto índice de esforço e baixa recompensa no trabalho. A literatura não há um consenso sobre presença ou ausência do estresse na equipe de enfermagem, fato que demonstra que outras variáveis (e não só o trabalho) podem estar interferindo e que o estresse realmente é multifatorial. Em uma unidade de internação especializada verificou-se que somente $22 \%$ dos profissionais da enfermagem possuíam estresse ocupacional. ${ }^{15}$

Uma investigação realizada com 2421 profissionais da saúde da atenção básica na Bahia demonstrou que apenas $32,2 \%$ apresentavam estresse psicossocial. Estes trabalhadores 
11 | Macedo ABT, Antoniolli L, Dornelles TM, Hansel LA, Tavares JP, Souza SBC

referiram baixo esforço e baixo excesso de comprometimento com o trabalho. O autor relacionou este resultado ao pouco tempo de trabalho no setor, sugerindo que o estresse psicossocial possui relação com o tempo de exposição ao agente estressor. ${ }^{16}$

Achados semelhantes ao desta pesquisa foram encontrados em trabalhadores de pronto socorro e terapia intensiva, locais onde o estresse é visto como um problema usual no ambiente hospitalar. Nestas investigações, o aparecimento do estresse está relacionado às dificuldades no relacionamento interpessoal e trabalho em equipe, sofrimento e morte dos pacientes e insatisfação com o trabalho. ${ }^{17-18}$

Acredita-se que na população estudada o aparecimento do estresse está relacionado à modificação na complexidade do cuidado hospitalar, ao aumento da gravidade dos pacientes que procuram atendimento em nível terciário e à realização de múltiplas tarefas, fatores que acabam gerando sobrecarga de trabalho. A avaliação do grau de dependência dos pacientes para os cuidados de enfermagem na instituição do estudo demonstrou um aumento do número de pacientes com cuidados semi-intensivos e intensivos. Em 2003, 68\% dos pacientes hospitalizados possuíam necessidade de cuidados mínimos. Já em 2014, 66,7\% dos pacientes necessitavam de cuidados semi-intensivos e $27 \%$ de cuidados intensivos, demonstrando alta dependência da equipe de enfermagem para as necessidades humanas básicas. ${ }^{12} \mathrm{O}$ risco para instabilidade hemodinâmica é outro fator que pode estar relacionado ao estresse da equipe, desencadeado pela modificação do perfil e gravidade da clientela, o que dificulta a organização e a rotina no turno de trabalho. ${ }^{7}$

Os maiores níveis de estresse foram encontrados nos trabalhadores alocados no noturno. O efeito do trabalho em turnos é um tema amplamente estudado, pois sabe-se que está intimamente ligado ao adoecimento do trabalhador. O quadro de pessoal à noite é reduzido devido à crença de que os pacientes dormem durante todo turno e, portanto, solicitam menos a equipe de enfermagem. Todavia, esta não parece ser a realidade deste setor, devido à gravidade dos pacientes. Estes exigem o mesmo tipo de cuidado de enfermagem durante as $24 \mathrm{~h}$ semelhante, 
Estresse psicossocial e resiliência: um estudo em profissionais da enfermagem | 12

com exames, procedimentos, cuidados de higiene e conforto, entre outros. O número reduzido de profissionais, aliado à alta demanda de trabalho, necessidade de maior atenção para as tarefas e até pelo fato de trabalhar em um período diferente do seu cronotipo biológico, pode estar desencadeando maiores níveis de estresse psicossocial nos profissionais do noturno. ${ }^{19}$

No modelo utilizado, o excesso de comprometimento com o trabalho é considerado um fator que pode interagir com o desequilíbrio esforço-recompensa, potencializando efeitos nocivos à saúde e ao bem-estar. Neste sentido, identificou-se uma correlação positiva e moderada entre excesso de comprometimento e estresse psicossocial, moderada correlação positiva entre esforço e excesso de comprometimento e baixa correlação negativa entre estresse e recompensa. Estes dados significam que, conforme aumenta o comprometimento, aumentará o estresse e a percepção de esforço no trabalho.

Estudo realizado com profissionais da saúde em São Paulo demonstrou que indivíduos com maior intensificação do excesso de comprometimento com o trabalho apresentam mais implicação na capacidade para o trabalho do que os demais trabalhadores. O autor entende que o excesso de comprometimento é um fator individual de busca excessiva pela realização e pelo desempenho no trabalho, podendo ser intensificado pelas pressões existentes no ambiente laboral. ${ }^{20}$ Nos profissionais que atendem pacientes com GMR, o excesso de comprometimento passa pelo sentimento de suprir todas as demandas dos pacientes sem deixar pendências, em um curto período. Esse sentimento, muitas vezes, pode estar ligado às questões históricas da profissão, pela vocação esperada dos profissionais da enfermagem. ${ }^{21}$

Observou-se baixos escores de resiliência na maioria dos profissionais da enfermagem da amostra, havendo uma correlação positiva, porém fraca, com o estresse psicossocial. A resiliência ainda é um construto pouco investigado nos profissionais da enfermagem no Brasil. Sabe-se que indivíduos com baixa resiliência possuem maior predisposição ao desenvolvimento de sintomas de estresse e ao burnout, podendo também potencializar o aparecimento de 
13 | Macedo ABT, Antoniolli L, Dornelles TM, Hansel LA, Tavares JP, Souza SBC

sintomas de outras patologias. Este fator pode explicar o elevado absenteísmo apresentado por este grupo de trabalhadores. ${ }^{18}$

Uma investigação realizada com profissionais da enfermagem do Maranhão demonstrou que $80 \%$ possuíam altos níveis de resiliência, com maiores escores nos trabalhadores com mais idade. ${ }^{22}$ Com a evolução na investigação da resiliência no trabalho, atualmente já é possível identificar pontos com necessidade de melhorias e fatores de proteção. Aprimorar a motivação para o trabalho, os relacionamentos interpessoais e o trabalho em equipe podem ser fatores de proteção para o trabalhador. ${ }^{6}$

O presente estudo não detectou diferença significativa no estresse e no escore de resiliência entre as categorias profissionais. Resultados diferentes foram encontrados em outros estudos que demonstraram que os enfermeiros de unidades de internação não apresentavam respostas fisiológicas significativas ao estresse e $^{17,23}$ e os técnicos se apresentavam mais resilientes do que os enfermeiros. ${ }^{18}$ Estes achados demonstram que os fatores estressores que atingem a categoria de nível médio também podem estar afetando os profissionais de nível superior.

A promoção da resiliência possibilita a redução dos impactos do estresse ocupacional nos trabalhadores da enfermagem, mesmo em situações de sobrecarga física e emocional. A literatura aponta como alternativa para construção da resiliência individual o autoconhecimento, o foco na fé e na esperança, e o exercício diário de driblar pensamentos negativistas e extremamente críticos. Para o aprimoramento da resiliência no trabalho, o fortalecimento das relações interpessoais e do trabalho em equipe. ${ }^{24}$

\section{Conclusão}

Este estudo identificou que a maioria dos profissionais do setor apresentavam estresse psicossocial, predominantemente na equipe do noturno. Também foi constatado percepção de alto esforço despendido para execução das tarefas do labor, baixa recompensa por parte da instituição, excesso de comprometimento com o trabalho e baixos escores de resiliência. 
Estresse psicossocial e resiliência: um estudo em profissionais da enfermagem | 14

Verificou-se relação do estresse psicossocial com o excesso de comprometimento e baixa correlação com a resiliência.

Esses dados são de extrema relevância, uma vez que esta combinação pode estar potencializando adoecimento nestes trabalhadores. Desta forma, percebe-se a necessidade de pesquisar e desenvolver estratégias para fortalecer a resiliência da equipe de enfermagem, melhorando suas capacidades de enfrentamento das situações estressantes inerentes aos elevados percentuais de risco para estresse psicossocial identificados na amostra.

Entende-se que uma limitação desta pesquisa foi que este é o único setor único setor da instituição a atender exclusivamente este tipo de paciente constituindo um número pequeno de trabalhadores. Contudo, em decorrência do crescente número de pacientes com germes multirresistentes, este estudo poderá contribuir para a implantação de novos setores exclusivos, já pensando em medidas na organização do trabalho que venham a auxiliar na redução do risco para estresse psicossocial e desenvolver a resiliência. Também poderá servir como embasamento e sensibilização para novas pesquisas na área, visto a carência de estudos relacionados a temática.

\section{Referências}

1. Goh J, Pfeffer J, Zenios S. Exposure to harmful workplace practices could account for inequality in life spans across different demographic groups. Health Aff [Internet]. 2015 [acesso em 2018 jul 10];34(10):1761-8. Disponível em: http://www.ncbi.nlm.nih.gov/pubmed/26438754

2. Moreira IJB, Horta JA, Duro LN, Chaves J, Jacques CS, Martinazzo K, et al. Aspectos psicossociais do trabalho e sofrimento psíquico na estratégia de saúde da família. Rev Epidemiol Controle Infecção [Internet]. 2017 [acesso em 2018 jul 10];7(1):2238-3360. Disponível em: https://online.unisc.br/seer/index.php/epidemiologia/article/viewFile/6927/5486

3. Siegrist J, Wege N, Pühlhofer F, Wahrendorf M. A short generic measure of work stress in the era of globalization: effort-reward imbalance. Int Arch Occup Environ Health [Internet]. 2009 [acesso em 2019 abr 03];82(8):1005-13. Disponível em: http://link.springer.com/10.1007/s00420-008-0384-3 
4. Lee TY, Cheung CK, Kwong WM. Resilience as a positive youth development construct: a conceptual review. ScientificWorldJournal [Internet]. 2012 [acesso em 2019 ago 28];12:1-9. Disponível em: http://www.hindawi.com/journals/tswj/2012/390450/

5. McEwen K. Building personal resilience as a geoscientist. Applied Earth Science (Trans Inst Min Metall B) [Internet]. 2012 [acesso em 2019 set 07];121:155-62. Disponível em: http://workingwithresilience.com.au/wp-content/uploads/2016/04/GeoscienceResilienceArticle.pdf

6. Sousa VFS, Araújo TCCF. Estresse ocupacional e resiliência entre profissionais de saúde. Psicol Ciênc Prof [Internet]. 2015 [acesso em 2019 jun 09];35(3):900-15. Disponível em: https://dialnet.unirioja.es/servlet/articulo?codigo $=6122628$

7. Martinez MC, Latorre MRDO, Fischer FM. Estressores afetando a capacidade para o trabalho em diferentes grupos etários na enfermagem: seguimento de 2 anos. Ciênc Saúde Colet [Internet]. 2017 [acesso em 2018 nov 04];22(5):1589-600. Disponível em: http://www.scielo.br/scielo.php?script=sci_arttext\&pid=S1413-81232017002501589\&lng=pt\&tlng=pt

8. Graça CC, Zagonel IPS. Estratégias de coping e estresse ocupacional em profissionais de enfermagem: revisão integrativa. Espaç Saúde [Internet]. 2019 [acesso em 2020 jan 14];20(2):67-77. Disponível em: http://espacoparasaude.fpp.edu.br/index.php/espacosaude/article/view/622/pdf

9. Silva SM, Borges E, Abreu M, Queirós C, Baptista PCP, Felli VEA. Relação entre resiliência e burnout: promoção da saúde mental e ocupacional dos enfermeiros. Rev Port Enferm Saúde Mental [Internet]. 2016 [acesso em 2020 jan 14];(16):41-8. Disponível em: http://www.scielo.mec.pt/scielo.php?script=sci_arttext\&pid=S1647-21602016000300006

10. Stumpfs DJ, Souza SBC, Konkewicz LR, Lovatto CG, Silva CF, Farias CF, et al. The impact of a single ward for cohorting patients with infection due to multidrug-resistant organisms. Infect Control Hosp Epidemiol [Internet]. 2013 [acesso em 2018 maio 16];34(08):864-5. Disponível em: https://www.cambridge.org/core/product/identifier/S0195941700033592/type/journal_article

11. Macedo ABT, Junges M, Mello DB, Lovatto CG, Souza SBC. Unidade para Portadores de Germes Multirresistentes: elaboração de um protocolo de atendimento de pacientes. Rev Enferm Atual In Derme [Internet]. 2017 [acesso em 2018 maio 16];83:61. Disponível em: https://revistaenfermagematual.com.br/index.php/revista/issue/view/12

12. Macedo ABT, Souza SBC, Funcke LB, Magalhães AMM, Riboldi CO. Sistematização de um instrumento de classificação de pacientes em um hospital universitário. REME Rev Min Enferm [Internet]. 2018 [acesso em 2019 maio 16];22:e-1152. Disponível em: http://www.reme.org.br/artigo/detalhes/1285

13. Macedo ABT. Avaliação da carga de trabalho, estresse psicossocial e resiliência nos profissionais de enfermagem em uma unidade de internação para adultos portadores de germes multirresistentes 
Estresse psicossocial e resiliência: um estudo em profissionais da enfermagem | 16

[dissertação]. Porto Alegre: Universidade Federal do Rio Grande do Sul; 2013 [acesso em 2019 maio 13]. Disponível em: https://www.lume.ufrgs.br/handle/10183/90196

14. Pesce RP, Assis SG, Avanci JQ, Santos NC, Malaquias JV, Carvalhaes R. Adaptação transcultural, confiabilidade e validade da escala de resiliência. Cad Saude Publica [Internet]. 2005 [acesso em 2018 jul 11];21(2):436-48. Disponível em: http://www.scielo.br/scielo.php?script=sci_arttext\&pid=S0102$311 X 2005000200010 \& \operatorname{lng}=p t \& \operatorname{lng}=p t$

15. Trettene AS, Andrade CS, Kostrich LM, Tabaquim MLM, Razera APR. Estresse em profissionais de enfermagem atuantes em um hospital especializado. Rev Enferm UFPE On Line [Internet]. 2016 [acesso em 2019 nov 22];10(12):4450-8. Disponível em: https://periodicos.ufpe.br/revistas/revistaenfermagem/article/view/11509/13387

16. Araújo TM, Mattos AIS, Almeida MMG, Santos KOB. Aspectos psicossociais do trabalho e transtornos mentais comuns entre trabalhadores da saúde: contribuições da análise de modelos combinados. Rev Bras Epidemiol [Internet]. 2016 [acesso em 2019 nov 22];19(3):645-57. Disponível em: http://www.scielo.br/scielo.php?script=sci_arttext\&pid=S1415-790X2016000300645\&lng=pt\&tlng=pt

17. Dalri RCMB, Silva LA, Mendes AMOC, Robazzi MLCC. Carga horária de trabalho dos enfermeiros e sua relação com as reações fisiológicas do estresse. Rev Latinoam Enferm [Internet]. 2014 [acesso em 2018 out 28];22(6):959-65. Disponível em: http://www.scielo.br/pdf/rlae/v22n6/pt_0104-1169-rlae-22-0600959.pdf

18. Medeiros PB, Assunção GA, Kniess JM, Dutra K, Preis LC, Lessa G . Resiliência e enfermagem: atributos adquiridos durante a profissão [Internet]. In: $3^{\circ}$ Forum de Direitos Humanos e Saúde Mental; 2017 jun 29-30; Florianópolis. Florianópolis: ABRASME; 2017 [acesso em 2018 jul 12]. Disponível em: http://www.direitoshumanos2017.abrasme.org.br/resources/anais/8/1491012697_ARQUIVO_resumo_resil iencia_forum(1).pdf

19. Oliveira B, De Martino MMF. Análise das funções cognitivas e sono na equipe de enfermagem nos turnos diurno e noturno. Rev Gaúch Enferm [Internet]. 2013 [acesso em 2018 out 28];34(1):30-6. Disponível em: http:/www.scielo.br/scielo.php?script=sci_arttext\&pid=S1983$14472013000100004 \& \operatorname{lng}=\mathrm{pt} \& \mathrm{t} \operatorname{lng}=\mathrm{pt}$

20. Martinez MC, Latorre MRDO, Fischer FM. Testando o modelo da casa da capacidade para o trabalho entre profissionais do setor hospitalar. Rev Bras Epidemiol [Internet]. 2016 [acesso em 2018 jul 12];19(2):403-18. Disponível em: http://www.scielo.br/scielo.php?script=sci_abstract\&pid=S1415790X2016000200403\&lng=en\&nrm=iso\&tlng=pt

21. Rodrigues AL, Barrichello A, Morin EM. Os sentidos do trabalho para profissionais de enfermagem: um estudo multimétodos. RAE Rev Adm Empresas [Internet]. 2016 [acesso em 2019 nov 23];56(2):192-208. Disponível em: http://bibliotecadigital.fgv.br/ojs/index.php/rae/article/view/60322 
22. Galvão APFC, Pinto JR. Resiliência em profissionais de enfermagem que atuam no setor de urgência e emergência psiquiátrica. J Manag Prim Health Care [Internet]. 2017 [acesso em 2019 nov 23];8(1):39-48. Disponível em: http://www.jmphc.com.br/jmphc/article/view/297/426

23. Guo YF, Luo YH, Lam L, Cross W, Plummer V, Zhang JP. Burnout and its association with resilience in nurses: a cross-sectional study. J Clin Nurs [Internet]. 2018 [acesso em 2018 jul 14];27(1-2):441-9. Disponível em: http://www.ncbi.nlm.nih.gov/pubmed/28677270

24. Ramalisa RJ, Du Plessis E, Koen MP. Increasing coping and strengthening resilience in nurses providing mental health care: empirical qualitative research. Health SA [Internet]. 2018 jul [acesso em 2018 jul 14]12;23:1094. Disponível em: https://www.ncbi.nlm.nih.gov/pubmed/31934384

\section{Autor correspondente}

Andréia Barcellos Teixeira Macedo

E-mail: abtmacedo@gmail.com

Endereço: Rua Ramiro Barcelos, 2350, Bairro Bom Fim, Porto Alegre, RS, Brasil

CEP: 90035-007

\section{Contribuições de Autoria}

\section{1 - Andréia Barcellos Teixeira Macedo}

Concepção e planejamento do projeto de pesquisa, obtenção, análise e interpretação dos dados, redação e revisão crítica.

\section{2 - Liliana Antoniolli}

Redação e revisão crítica.

\section{3 - Thayane Martins Dornelles}

Redação e revisão crítica.

\section{4 - Leandro Augusto Hansel}

Redação e revisão crítica.

5 - Juliana Petri Tavares

Redação e revisão crítica.

\section{6 - Sonia Beatriz Cocaro de Souza}

Concepção e planejamento do projeto de pesquisa, obtenção, análise e interpretação dos dados, redação e revisão crítica.

\section{Como citar este artigo}

Macedo ABT, Antoniolli L, Dornelles TM, Hansel LA, Tavares JP, Souza SBC. Estresse psicossocial e resiliência: um estudo em profissionais da enfermagem. Rev. Enferm. UFSM. 2020 [Acesso em: Anos Mês Dia]; vol.10 e25: 1-17. DOI:https://doi.org/10.5902/2179769235174 